\title{
RESEARCH HIGHLIGHT Panning for brain antigens in dural sinuses
}

\author{
Ania Bogoslowski (D) ${ }^{1}$ and Paul Kubes ${ }^{2}$ \\ Cell Research (2021) 31:607-608; https://doi.org/10.1038/s41422-021-00502-z
}

\begin{abstract}
Understanding immunosurveillance of the brain is key to developing strategies to treat and prevent neurodegenerative diseases with immune components. Rustenhoven et al. capture the intricacies of $T$ cell interactions with antigenpresenting cells in the dura, to explain exactly where and how surveillance at steady state occurs.
\end{abstract}

The immunosurveillance of the brain is just beginning to be understood. The brain was long thought to be an immune privileged site and while this remains highly debated, research in the last decade has shown that it is not quite so simple. There is communication between the brain tissue parenchyma and the immune system, as evidenced by many neuroimmune disorders. Much of the immune surveillance is carried out by populations of immune cells in the tissues surrounding the brain parenchyma, particularly in the meningeal lymphatic network. ${ }^{1}$

The importance of these surveillance immune cell populations in the dura has been shown under many conditions. The meningeal lymphatics are essential for the development of strong autoinflammatory responses in a mouse model of multiple sclerosis (MS), experimental autoimmune encephalomyelitis $(E A E){ }^{2}$ When the lymphatics were ablated, reactive T cells were reduced. In brain tumor models after metastasis, the brain lymphatics change their transcriptional phenotype. ${ }^{3}$ By disrupting the meningeal lymphatics, mice experienced overall worse outcomes due to a reduction in dendritic cell trafficking and reduced response to anti-PD-1 therapy. ${ }^{3}$ Enhanced expression of VEGF-C in the lymphatics promoted antitumor responses, through improved CD8 T cell priming and infiltration into tumor tissue. ${ }^{4}$ The antigen surveillance of the brain is not restricted to the dura, as patients with Parkinson's disease or Alzheimer's disease have circulating T cells specific for central nervous system (CNS) antigens. ${ }^{5,6}$

Even with this body of knowledge, what is still lacking is an understanding of the steady-state interactions between $T$ cells and antigen-presenting cells (APCs) and the structures where this occurs, to lead to initiation of disease. Rustenhoven et al. tackle this puzzle in their recent paper. ${ }^{7}$ Using a combination of singlecell RNA sequencing (scRNA-seq) and intravital microscopy, the findings in this paper build a basis for understanding the steadystate interactions that could lead to disease.

The study begins with a detailed spatial overview of immune cell populations that reside within the dural sinuses. Using mass cytometry CyTOF, the authors observed that at steady state the immune populations present in this tissue vary between old and young mice, specifically with an increase in T cell populations in aged mice. The focus was shifted to an equally detailed description of the stromal cells of the dural sinuses, using mainly scRNA-seq data complemented with whole-mount immunohistochemistry.
Rustenhoven et al. were able to combine these data to identify possible leukocyte interactions within sinus and lymphatic vasculature. Focusing on T cells, the authors tracked transferred $T$ cells in the dura, characterized their behavior and observed PSGL-1/VLA4/LFA1-dependent adhesion. When these three adhesion molecules were blocked, $T$ cell adhesion events were virtually eliminated. This observation offers a potential avenue for therapeutic development. In neuroimmune diseases, where the trafficking of CD4 T cells to the brain promotes inflammatory responses, employing an antibody or alternative inhibitor to block $T$ cell binding to sinus endothelium, could improve disease states. Importantly, the authors included human samples that showed the expression of VCAM, suggesting that human immunosurveillance occurs through a very similar mechanism. Interestingly, while the concept of selectins and integrins is similar to what is seen in lymph nodes (Fig. 1), the results vary in that rather than Lselectin and its ligands known to be important for naïve $T$ cell recruitment to these immune structures, in the case of dural sinuses, P-selectin and PSGL-1 are invoked for T cell homing. This is reminiscent of a study showing that P-selectin is important for thymic lymphocytic progenitor recruitment to thymus ${ }^{8}$ and the fact that P-selectin seems to reduce neuroinflammatory phenotypes albeit through a different P-selectin ligand, TIM-1.9

Not only did T cells interact with the endothelium, but they also exhibited the ability to migrate into the dura. GFP-expressing CD4 $T$ cells were found in the perisinual area of the dura 6 days after being intravenously transferred. In a parabiotic model, after 1.5 months of pairing, the T cells in this zone were $50 \%$ partnerderived. This clearly demonstrates that the dural sinuses are a major site of T cell surveillance from the blood stream.

To identify targets for the observed $T$ cell recruitment and retention, the authors turned to the scRNA-seq data of the meningeal populations to predict adhesiveness and identified several putative physical and signaling interactions, one of which was CXCL12-CXCR4. Furthermore, CXCL12 was increased in almost all stromal cell subtypes based on scRNA-seq and whole-mount immunohistochemistry. Functionally, PDGFR-specific CXCL12knockout mice had reduced $\mathrm{T}$ cell homing to the dura. It would be interesting to see whether patients who develop neuroimmune disease have increased CXCL12 expression, as this could promote more $\mathrm{T}$ cell recruitment at steady state, thus resulting in an imbalanced immune surveillance system and predisposing to disease. It is worth noting that this pathway may also function as a homing receptor back to bone marrow and has been targeted in WHIM syndrome (increased activity in CXCR4) in humans without adverse effects. ${ }^{10}$

Another observation that Rustenhoven et al. made is that the dura is a strategic site for immune surveillance due to the efflux of

\footnotetext{
${ }^{1}$ Department of Medical Genetics, Life Science Institute, University of British Columbia, Vancouver, BC, Canada and ${ }^{2}$ Snyder Institute of Infection, Immunity, and Inflammation, University of Calgary, Calgary, AB, Canada

Correspondence: Paul Kubes (pkubes@ucalgary.ca)
}

Published online: 6 May 2021 


\section{Lymph node}
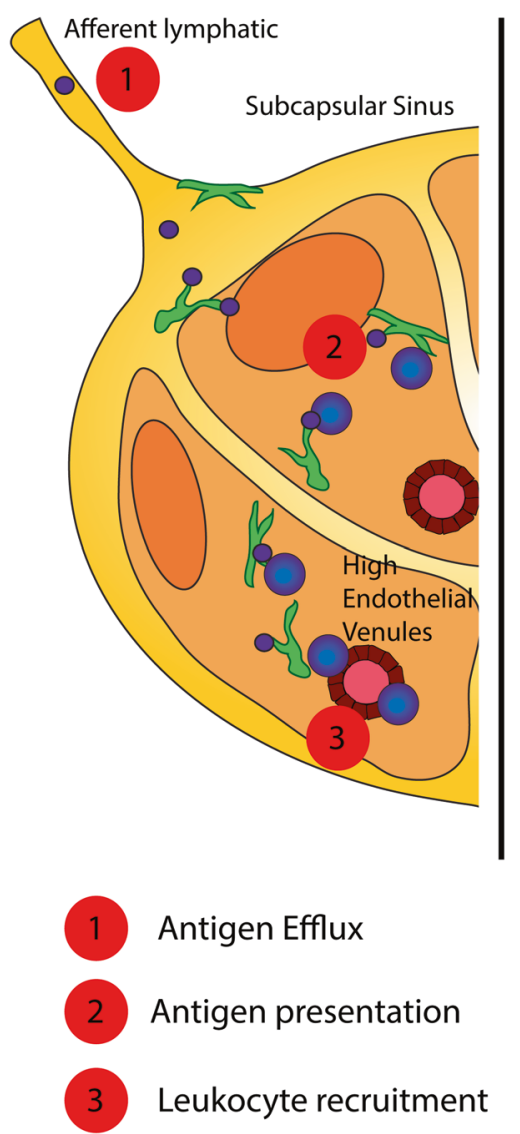

Antigen Efflux

Antigen presentation

Leukocyte recruitment

\section{Dura}
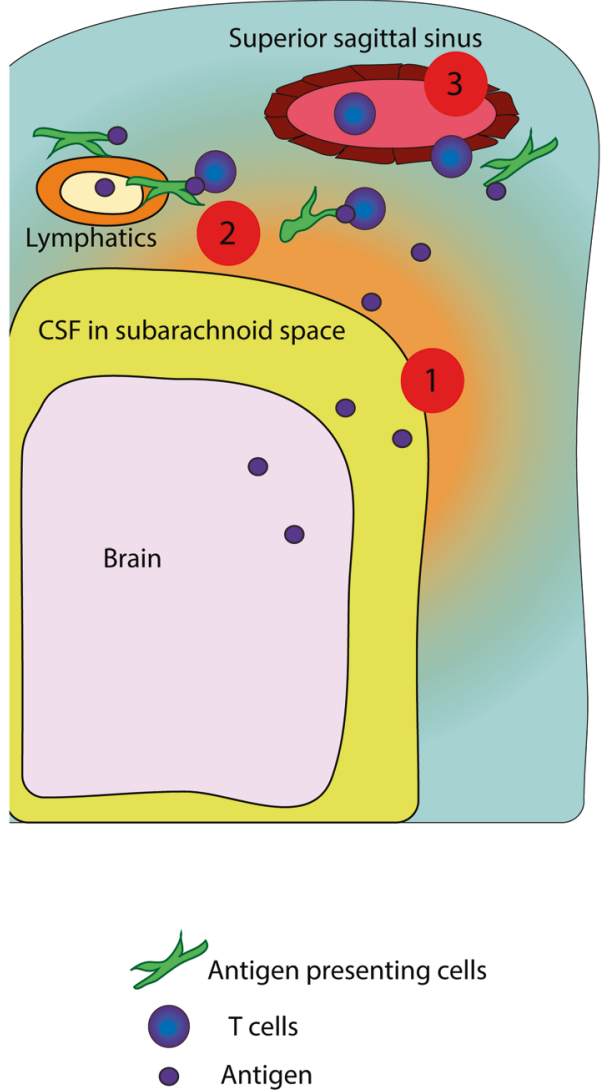

Antigen

Fig. 1 Similarities between antigen surveillance in the lymph node and the dural sinuses. In the lymph node, antigen enters through the afferent lymphatic vessels and is picked up by macrophages in the subcapsular sinus and dendritic cells in the parenchyma for antigen presentation. T cells enter through high endothelial venules, a process carefully controlled by L-selectin interactions. In the brain, antigen drains from the brain through the subarachnoid space and into the Dura. Here, antigen presenting cells pick up brain antigen and present it to $T$ cells which infiltrate through the superior sagittal sinus in a VLA4/LFA/PSGL-1 dependant manner.

cerebral spinal fluid (CSF) and the opportunity for sampling. This was demonstrated by using fluorescent tracers injected into the CSF and imaging of the dural sinuses. Thus the role of the dura is not just restricted to cell homing, but fluid from the CSF also filters through these structures. This means that APCs that reside in the tissue could act as antigen presenting cells. To determine whether this is the case, OT-II mice (with transgenic T cell receptors that recognize OVA protein) were transferred to mice that had received an injection of fluorescently-labeled OVA into the CSF. In these mice, APCs in the dural sinuses would capture the OVA protein and this resulted in a concomitant accumulation of OT-II T cells.

Finally, the authors applied these observations to a model of MS, induced by injection of MOG peptide to induce $T$ cell activation. They found that MOG-specific T cells accumulate in the dural sinuses, as opposed to non-sinus areas. This demonstrated that $\mathrm{T}$ cell surveillance of the CNS is important in the disease condition as well as steady state.

The more neuroinflammatory diseases are studied, the more akin they seem to be to autoimmunity elsewhere in the body. $\mathrm{A}$ major part missing in the field was where do T cells, antigen, and APCs meet and this work unveils that the peripheral immune system learns about what is happening in the brain at dural sinuses. Like any good study, this work raises many new questions that will await answers. Whether there is an inevitable linkage to neuroinflammatory diseases with age and whether one can find a selective pathway to target will need further study.

\section{REFERENCES}

1. Louveau, A. et al. Nature 523, 337-41 (2015).

2. Louveau, A. et al. Nat. Neurosci. 21, 1380-1391 (2018).

3. Hu, X. et al. Cell Res. 30, 229-243 (2020).

4. Song, E. et al. Nature 577, 689-694 (2020).

5. Sulzer, D. et al. Nature 546, 656-661 (2017).

6. Monsonego, A. et al. J. Clin. Invest. 112, 415-422 (2003).

7. Rustenhoven, J. et al. Cell 184, 1000-1016 (2021).

8. Rossi, F. M. V. et al. Nat. Immunol. 6, 626-634 (2005).

9. Angiari, S. et al. Immunity 4, 542-553 (2014).

10. Dale, D. C. et al. Blood 118, 4963-4966 (2011). 